\title{
Real-time terahertz imaging through self-mixing in a quantum-cascade laser
}

\author{
M. Wienold, ${ }^{1,2, a)}$ T. Hagelschuer, ${ }^{1}$ N. Rothbart, ${ }^{1,2}$ L. Schrottke, ${ }^{3}$ K. Biermann, ${ }^{3}$ H. T. Grahn, ${ }^{3}$ \\ and H.-W. Hübers ${ }^{1,2}$ \\ ${ }^{1}$ Institute of Optical Sensor Systems, German Aerospace Center (DLR), Rutherfordstr. 2, 12489 Berlin, \\ Germany \\ ${ }^{2}$ Department of Physics, Humboldt-Universität zu Berlin, Newtonstr. 15, 12489 Berlin, Germany \\ ${ }^{3}$ Paul-Drude-Institut für Festkörperelektronik, Leibniz-Institut im Forschungsverbund Berlin e. V., \\ Hausvogteiplatz 5-7, 10117 Berlin, Germany
}

(Received 12 April 2016; accepted 22 June 2016; published online 7 July 2016)

\begin{abstract}
We report on a fast self-mixing approach for real-time, coherent terahertz imaging based on a quantum-cascade laser and a scanning mirror. Due to a fast deflection of the terahertz beam, images with frame rates up to several $\mathrm{Hz}$ are obtained, eventually limited by the mechanical inertia of the employed scanning mirror. A phase modulation technique allows for the separation of the amplitude and phase information without the necessity of parameter fitting routines. We further demonstrate the potential for transmission imaging. Published by AIP Publishing.

[http://dx.doi.org/10.1063/1.4955405]
\end{abstract}

Terahertz $(\mathrm{THz})$ imaging with quantum-cascade lasers (QCLs) has seen a large scientific interest in recent years. ${ }^{1} \mathrm{~A}$ sensitive but single-pixel detection approach is provided by the self-mixing effect in QCLs, i.e., its sensitivity to external optical feedback (EOF). The phase sensitivity of the effect has triggered the development of sophisticated techniques for the determination of the complex dielectric constant of reflective targets ${ }^{2,3}$ and three-dimensional target reconstructions. ${ }^{4}$ Typically, images are obtained by scanning the target in two dimensions via linear translation stages. While a high spatial resolution has been demonstrated, ${ }^{5,6}$ the acquisition time for a single frame is on the order of several minutes to hours in such a configuration. Image acquisition times on the order of $1 \mathrm{~s}$ have been demonstrated by using a fast scanning mirror and a He-cooled Ge:Ga bolometer with a short response time. ${ }^{7}$ However, the phase information is lost in such a configuration due to the incoherent detection. In this work, we show that exploiting the self-mixing effect allows for fast and phase-sensitive real-time imaging in an optical configuration with a scanning mirror.

The experimental setup is shown in Fig. 1. The optical path consists of the THz QCL mounted in a mechanical cryocooler (Ricor, model K535), ${ }^{8}$ two lenses for beam collimation and focusing, the scanning mirror for the deflection of the $\mathrm{THz}$ beam, a wire grid as a tunable attenuator, and the target. According to the geometry of the optics, the diameter of the field of view is limited by $D_{\mathrm{FOV}} \leq 4 f_{2} \alpha_{m}$ as well as $D_{\mathrm{FOV}} \leq D_{2}$, where $f_{2}$ and $D_{2}$ denote the focal length and diameter of lens 2 , respectively, and $\alpha_{m}=3^{\circ}$ the maximum deflection of the scanning mirror from its default position. For example, by using a lens with $D_{2}=76 \mathrm{~mm}$ and $f_{2}=250 \mathrm{~mm}$, targets up to about $50 \mathrm{~mm}$ in diameter can be imaged. Via the beam waist in the focal plane, the second lens limits also the obtainable spatial resolution, which eventually causes a trade-off between field of view and resolution. The employed QCL emits at $3.3 \mathrm{THz}$ and is based on the active-region design described in Ref. 9 and a standard

${ }^{\text {a)} E l e c t r o n i c ~ m a i l: ~ m a r t i n . w i e n o l d @ d l r . d e ~}$ single-plasmon Fabry-Pérot cavity $\left(0.1 \times 1.2 \mathrm{~mm}^{2}\right)$. In its simplest configuration, the setup requires just a constant current source and fast data acquisition (DAQ) device to record the self-mixing signal, i.e., the ac component of the QCL terminal voltage. However, the acquired images then contain a mixture of amplitude and phase information, which is usually difficult to interpret. We circumvent this obstacle by a phase-modulation technique. The basic idea is to vary the optical phase over an interval of $2 \pi$ by exploiting the dependence of the laser frequency on the driving current. The amplitude information is obtained by integrating the phasemodulated signal; the factor containing the self-mixing phase cancels out. For modulation purpose, the setup contains a function generator (Agilent, 33120A) connected to the modulation input of the current source (Lightwave, LDX3232). It further contains a voltage divider circuit and a differential amplifier (Stanford Research Systems, SRS 560) to cancel the part of the voltage modulation which originates directly from the current-voltage characteristics and is not related to EOF. The main purpose of this basic compensation scheme is to avoid saturation of the analog-to-digital converter.

Two characterizing quantities for self-mixing measurements are the feedback parameter $C$ and the linewidth enhancement factor $\alpha$. The former mainly characterizes the level of back-reflected light and is determined by the optical setup as well as the QCL cavity. The latter characterizes the interplay between the gain and the effective index of the cavity mode and is governed by the active-region design as well as the operating parameters of the QCL. Note that $\alpha$ can also become negative according to its definition, ${ }^{10}$ which was confirmed for mid-infrared QCLs. ${ }^{11}$ The self-mixing equations as derived from the Lang-Kobayashi model for a semiconductor laser under stationary feedback ${ }^{2,12,13}$ are given by

$$
\begin{gathered}
\phi_{0}-\phi_{\mathrm{EOF}}=C \sin \left[\phi_{\mathrm{EOF}}-\arctan (\alpha)\right], \\
V=V_{0}+V_{\mathrm{sm}}=V_{0}+\beta \cos \left(\phi_{\mathrm{EOF}}\right) .
\end{gathered}
$$

Equation (1) describes the impact of EOF on the interferometric phase $\phi_{\mathrm{EOF}}=2 \pi \nu \tau_{\mathrm{ext}}$, where $\nu$ denotes the laser frequency 


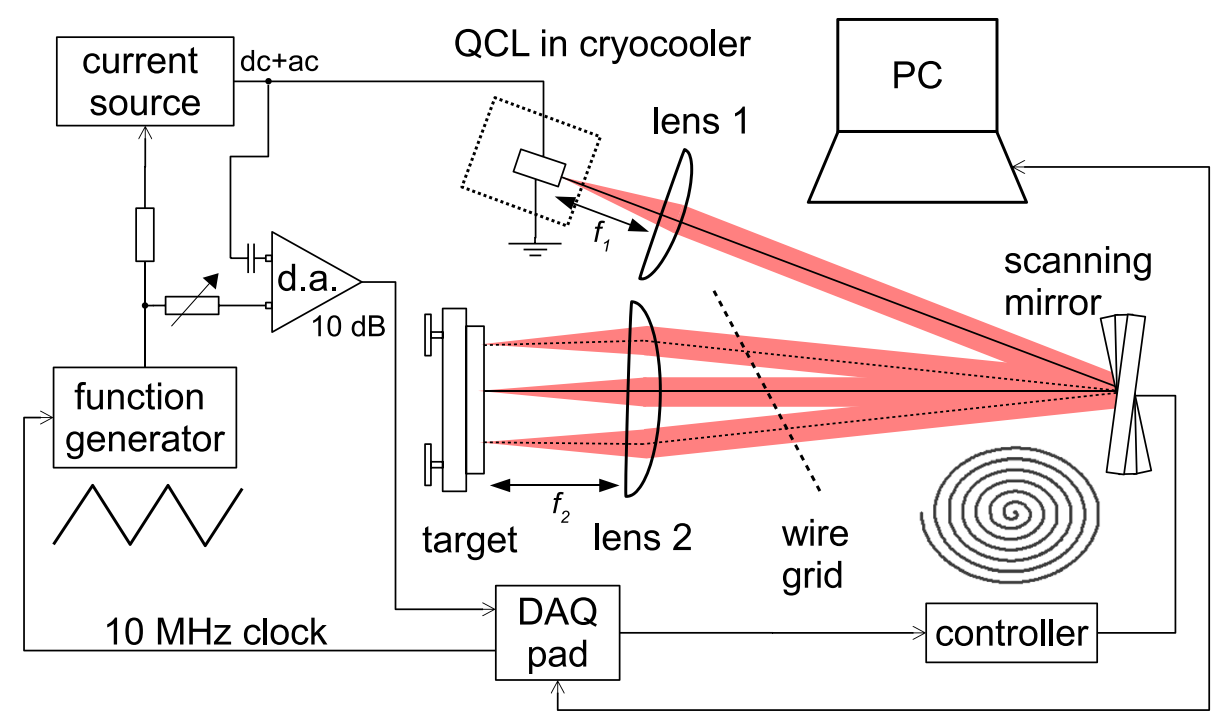

FIG. 1. Schematic of the experimental setup for self-mixing imaging. The $\mathrm{THz}$ beam emitted by the QCL is scanned across the target in two dimensions by the scanning mirror. d.a.: differential amplifier; focal length of lenses 1 and 2: $f_{1}=92 \mathrm{~mm}$ and $f_{2}=140-250 \mathrm{~mm}$. The optical path length is $50-80 \mathrm{~cm}$.

and $\tau_{\text {ext }}$ is the EOF delay with respect to the laser facet. $\phi_{0}=$ $2 \pi \nu_{0} \tau_{\text {ext }}$ is the interferometric phase of the unperturbed system at frequency $\nu_{0}$. Equation (2) describes the modulation of the QCL operating voltage by EOF, which is a consequence of the modulation of the gain. $V_{\mathrm{sm}}$ denotes the component due to self-mixing and $V_{0}$ is the operating voltage without feedback. The coefficient $\beta$ is given by $\beta=\beta_{0} C / \sqrt{1+\alpha^{2}}$, where $\beta_{0}$ is a constant. The feedback parameter $C$ is given by

$$
C=\varepsilon \frac{\left(1-R_{c}\right)}{\sqrt{R_{c}}} \frac{L_{\mathrm{ext}}}{n_{c} L_{c}} \sqrt{R_{t}\left(1+\alpha^{2}\right)}=C_{0} \sqrt{R_{t}},
$$

where $\varepsilon, R_{c}$, and $R_{t}$ denote the coupling efficiency, the cavity, and the target power reflectivity, respectively, $L_{c}$ and $L_{\text {ext }}$ are the length of the QCL and the external cavity, respectively, and $n_{c}$ is the modal effective index of the QCL.

We used two complementary methods to determine the maximum level of feedback $C_{0}$ as well as $\alpha$ under similar conditions as for imaging. In the first configuration, the optical phase is altered by changing the QCL driving current. ${ }^{14} \mathrm{~A}$ mechanical chopper and a lock-in amplifier are used to record the self-mixing voltage, while the scanning mirror is held in its default position. The corresponding optical arrangement is depicted in Fig. 2(a). The beam is attenuated by a tunable wire grid in order to avoid discontinuities in the recorded signal, which occur at higher feedback levels and are difficult to analyze. The experimental results are shown in Fig. 2(b), where the solid line refers to the result of a simulation based on Eqs. (1) and (2) with parameters $\alpha=-0.9$ and $C=2.6$ (corresponding to $C=3.1$ without attenuation). For the calculation, we assumed a linear dependence of the frequency $\nu_{0}$ on the driving current with a slope of $+17 \mathrm{MHz} / \mathrm{mA}$. The small walk-off between the periodicity of the calculated and the measured signal is caused by deviations from the linear dependence. The second method exploits the modulation of the optical phase by the displacement of a plane mirror mounted on a voice coil ${ }^{15,16}$ as illustrated in Fig. 2(c). The measured self-mixing signal is plotted in Fig. 2(d) as a function of time for a sinusoidal oscillation of the voice coil. The solid line corresponds to the result of a calculation with $C=3.1$ and $\alpha=-0.9$. We found that non-zero values of $\alpha$ cause the formation of different oscillation amplitudes for regions, which are related to the forward and backward movements of the mirror. From the results of both experiments, we estimate $C_{0}=3.1 \pm 0.2$ and $\alpha=-0.9 \pm 0.3$. The level of feedback corresponds to the moderate feedback regime, ${ }^{13}$ for which the system can exhibit a bistable behavior and hysteresis. For imaging purposes, the wire grid is used to attenuate the feedback until images free of distortions are obtained. While small positive values ( $\alpha \approx 0.5$ ) have been reported for a $\mathrm{THz}$ QCL with a bound-to-continuum active region, ${ }^{14} \alpha$ significantly deviates from that value for the present QCL, which is based on an active region with phonon-assisted electron injection. 9

For a more detailed explanation of the applied phase modulation scheme for imaging, we start from Eq. (1). In the limit of weak feedback, it can be approximated by the algebraic form $\phi_{0}-\phi_{\mathrm{EOF}} \approx C \sin \left[\phi_{0}-\arctan (\alpha)\right]$. The expression for the self-mixing voltage then becomes

$$
V_{\mathrm{sm}}=\beta \cos \left\{\phi_{0}-C \sin \left[\phi_{0}-\arctan (\alpha)\right]\right\} .
$$
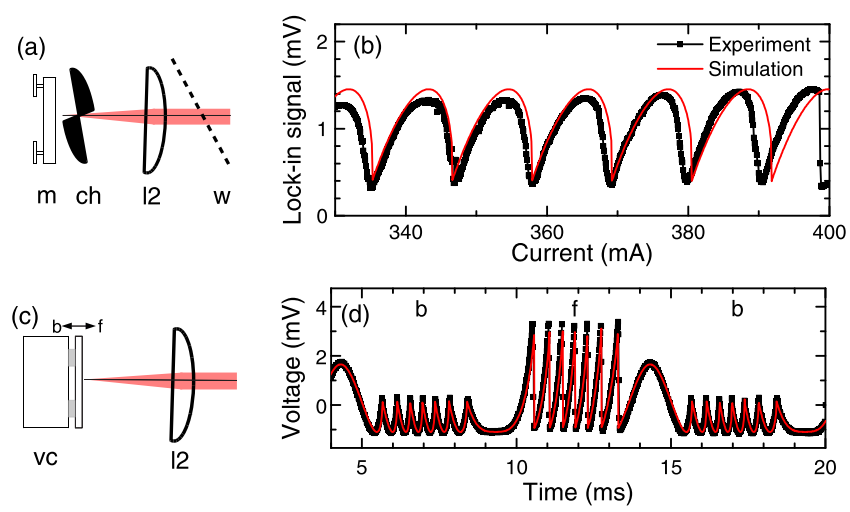

FIG. 2. (a) Optical configuration for determination of the feedback parameters $C$ and $\alpha$. m: plane mirror; ch: chopper; 12 : lens with $f_{2}=250 \mathrm{~mm}$; w: wire grid; and $L_{\text {ext }}=78 \mathrm{~cm}$. (b) Self-mixing signal as a function of the current for configuration (a). Simulation results are for $C=2.6$ and $\alpha=-0.9$. (c) Optical configuration with voice coil (vc). (d) Results for a sinusoidal modulation of the voice coil at $100 \mathrm{~Hz}$ with an amplitude of $180 \mu \mathrm{m}$. The QCL is operated in a single-mode regime at $3.32 \mathrm{THz}(370 \mathrm{~mA}, 46 \mathrm{~K})$. Simulation results are for $C=3.1$ and $\alpha=-0.9$. f: forward and b: backward movement of the mirror. 
We now employ a saw-tooth current modulation such that $\phi_{0}$ changes linearly by $2 \pi$ over one modulation cycle. Integration over one cycle and dividing by $2 \pi$ yields the averaged self-mixing voltage

$$
\left\langle V_{\mathrm{sm}}\right\rangle=\beta_{0} \frac{C}{1+\alpha^{2}} \mathrm{~J}_{1}(C) \approx \frac{\beta_{0}}{2} \frac{C^{2}}{1+\alpha^{2}}=p R_{t} .
$$

Here, $\mathrm{J}_{1}(C)$ denotes the Bessel function of the first kind, which can be approximated by $0.5 C$ for values of $C<1$. Constant prefactors are eventually subsumed in the coefficient $p$. For the integrated total voltage $\langle V\rangle$, we have to add an offset term due to $V_{0}$ in Eq. (2), which yields $\langle V\rangle=\left\langle V_{0}\right\rangle+p R_{t}$. The reconstruction of the interferometric phase $\phi_{0}$ requires two data sets, for which the difference in phase due to the frequency change is $\phi_{+}-\phi_{-}=\pi$. Defining the normalized quantities $N_{ \pm}=V_{\mathrm{sm}}\left(\phi_{ \pm}\right) / \beta$, one obtains $2 \phi_{0}\left(I_{-}\right)=2 \phi_{-}=\arccos \left(N_{+}\right)$ $+\arccos \left(-N_{-}\right)$, where $\phi_{0}\left(I_{-}\right)$is the interferometric phase for the laser frequency at the current value $I_{-}$. In practice, $N_{+/-}$ can be obtained from the acquired signals $V\left(I_{+} /-\right)$by normalizing the expression $\left(V-V_{0}\right) / \sqrt{\langle V\rangle-\left\langle V_{0}\right\rangle}$ to the interval $[-1,+1]$, which is applicable if the phase shift inside the field of view exceeds $\pi$. The offset terms $V_{0}$ and $\left\langle V_{0}\right\rangle$ can be obtained from a background measurement or from a region in the field of view with vanishing reflectivity (where the interferometric phase is undefined).

In order to illustrate the potential of the phasemodulation scheme, Fig. 3 depicts the result of simulations based on Eqs. (1) and (2), i.e., without the approximation made for Eqs. (4) and (5). Figure 3(a) depicts the assumed power reflectivity and the interferometric phase $\phi_{0}$ for a hypothetical target with a width of $20 \mathrm{~mm}$ and a tilt of $1^{\circ}$ with respect to the focal plane. The power reflectivity of the target follows a $W$ modulation. Due to the cosine in Eq. (2), the phase is mapped into the interval $[0, \pi]$. Figure $3(\mathrm{~b})$ depicts the resulting self-mixing signal for a fixed value of the current, which does not allow for a distinction between amplitude and phase information. The reconstructed power reflectivity and interferometric phase for a saw-tooth current modulation are shown in Figs. 3(c) and 3(d) for maximum feedback levels of $C_{0}=1$ and 3.1, respectively. While the reconstruction of the power reflectivity tolerates larger levels of feedback, the reconstruction of the interferometric phase is already disturbed for $C_{0}=1$. Hence, feedback levels
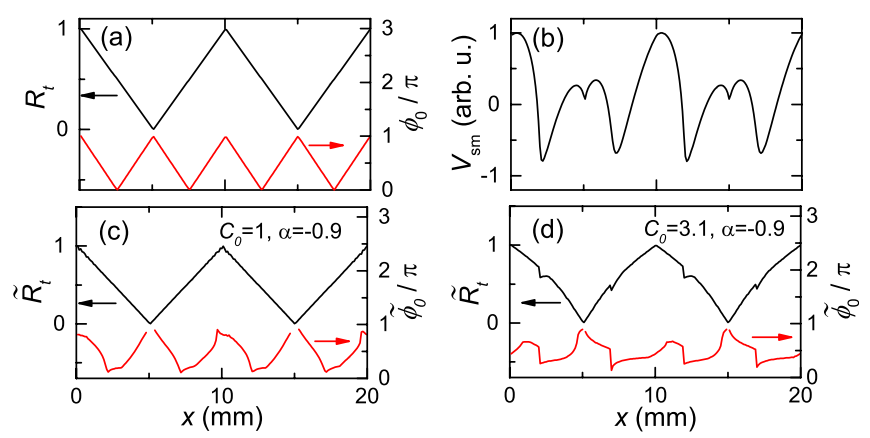

FIG. 3. (a) Assumed power reflectivity $R_{t}$ and interferometric phase $\phi_{0}$ for a simulated target as a function of the lateral position $x$. (b) Calculated selfmixing voltage for a fixed current value and $C_{0}=1$. (c) and (d) Reconstructed power reflectivity $\tilde{R}_{t}$ and interferometric phase $\tilde{\phi}_{0}$ for $C_{0}=1$ and $C_{0}=3.1$, respectively.
$C_{0} \ll 1$ are required for a reconstruction of $\phi_{0}$ on a quantitative level.

A typical experimental configuration uses a $20 \mathrm{kHz}$ triangular current modulation at a $500 \mathrm{kHz}$ data acquisition rate, which allows for a sampling of 25 data sets within one modulation cycle. The QCL is operated in a single-mode regime at a temperature of $48 \mathrm{~K}$ and a current of $370 \mathrm{~mA}$ ( $40 \mathrm{~mA}$ above threshold). During the measurement, the scanning mirror moves continuously. The mirror position and the self-mixing voltage are recorded synchronously. Figure 4(a) depicts a self-mixing image of the backside of a German 50Cent coin recorded by a spiral scan without current modulation ( $4 \mathrm{~s}$ recording time). In this configuration, fringes due to the non-planar phase front of the scanned $\mathrm{THz}$ beam and the tilt of the sample are clearly visible. In order to obtain fringe-free images, the amplitude of the current modulation is now increased until the fringes in the phase-integrated image disappear (corresponding to a modulation amplitude of about $10-15 \mathrm{~mA})$. The result is depicted in Fig. 4(b), showing the details of the coin free of disturbances. Figure 4(c) depicts the same target in the phase contrast mode. Fringes due to the change of the interferometric phase are now more clearly visible than in Fig. 4(a). For the calculation of the normalized quantities $N_{+/-}$, we assumed $\langle V\rangle$ as constant. We found that a phase contrast similar to Fig. 4(c) is already obtained by the difference of the two data sets recorded for $I_{+/-}$(not shown).

In practice, the frame rate of the setup is limited by the mechanical inertia of the scanning mirror; beyond a maximum acceleration, nonlinearities result in irregular trajectories. For imaging, we typically use an Archimedean spiral with a constant angular acceleration given by $\left(\alpha_{x}+i \alpha_{y}\right) / \alpha_{m}$ $=(f t)^{2 / 3} \exp \left[2 \pi i(f t)^{2 / 3} N\right]$. Here, $\alpha_{x}$ and $\alpha_{y}$ denote the mirror deflection in $x$ and $y$ directions, respectively, $f$ is the frame rate, $t$ is the time, and $N$ is the number of cycles given by $N=f^{-1} \sqrt{a_{m} / \alpha_{m}}$, with $a_{m}$ being the angular acceleration. At a sampling rate of $500 \mathrm{kHz}$, the amount of raw data which has to be processed is $3 \mathrm{MB} / \mathrm{s}$. This is realized in real-time via an optimized LabVIEW routine.

Figure 4(d) shows a series of images recorded for frame rates of $0.5-2 \mathrm{~Hz}$. While at low frame rates the spatial resolution is limited by the beam waist in the focal plane, the resolution at an increasing frame rate becomes limited by the number of possible cycles along the spiral trajectory, i.e., by the maximum angular acceleration of the scanning mirror. Without interpolation, circular images with up to 300 pixels in diameter $\left(71 \times 10^{3}\right.$ pixels per image) are obtained for a frame rate of $0.25 \mathrm{~Hz}$. This number reduces to 200 $\left(31 \times 10^{3}\right)$ for $0.5 \mathrm{~Hz}$ and $75\left(4.4 \times 10^{3}\right)$ pixels for $2 \mathrm{~Hz}$. Higher frame rates are possible accepting a further reduction of the resolution or a smaller field of view.

In Fig. 4(e), the power spectrum of the self-mixing and the background signal are compared for a frame rate of $0.25 \mathrm{~Hz}$. For the background measurement, the target has been replaced by an absorber (Eccosorb). The various spikes in the background signal are mainly due to vibrations of the cryocooler $(45 \mathrm{~Hz}$ and higher harmonics) and an electrical pickup of the power line frequency $(50 \mathrm{~Hz}$ and higher harmonics). Most of the spikes disappear when the cooler is switched off. Since the self-mixing signal in Fig. 4(e) is on 

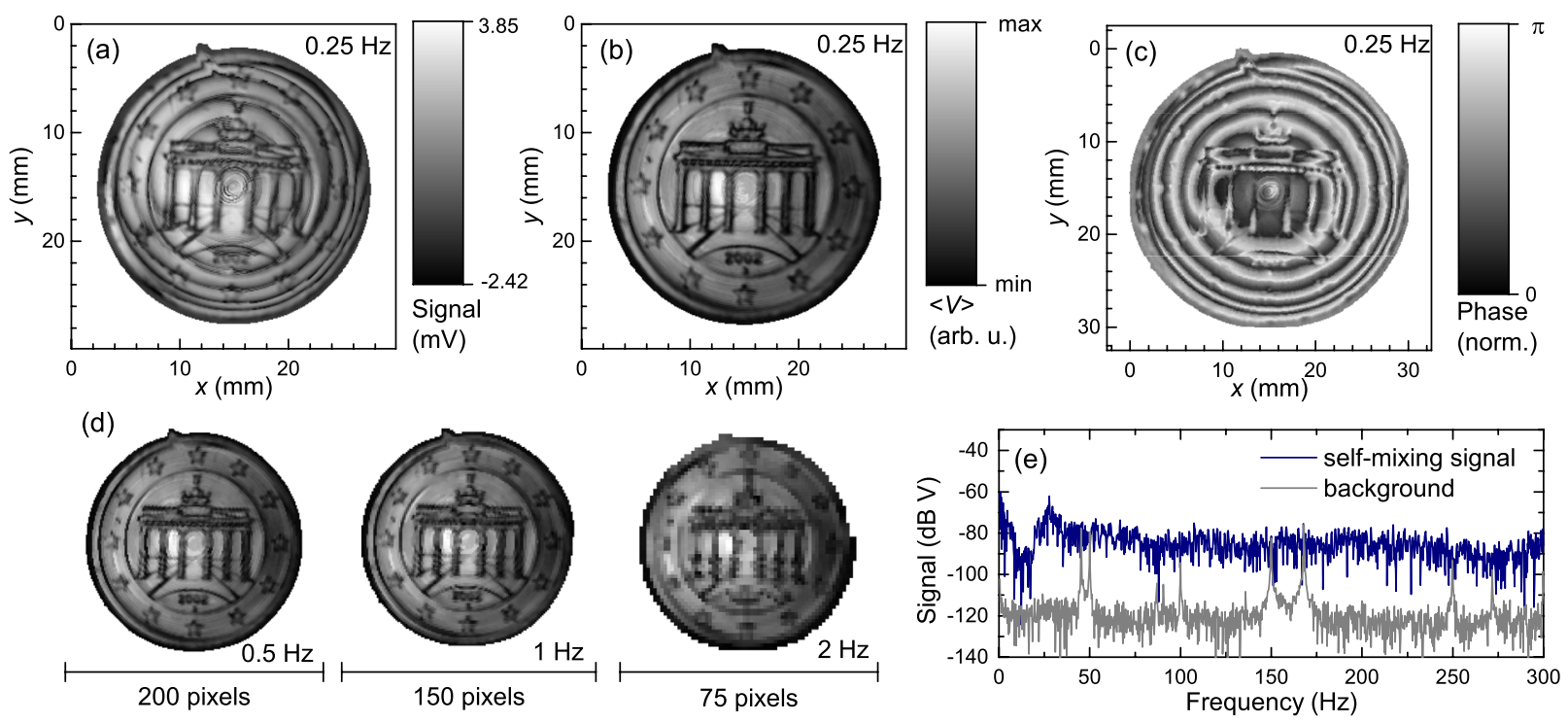

FIG. 4. (a) Self-mixing image of the backside of a German 50-cent coin using a spiral scan without current modulation. The recording time was $4 \mathrm{~s}$ $\left(f_{2}=140 \mathrm{~mm}\right.$ ). (b) Amplitude contrast and (c) phase contrast self-mixing image of the same target using a triangular current modulation with $20 \mathrm{kHz}$. (d) Series of the phase-integrated images for frame rates of $0.5-2 \mathrm{~Hz}$ (single-frame images). The artifact in the upper left part of each image stems from the position sensors of the scanning mirror. (e) Power spectrum of the self-mixing and background signal corresponding to $0.25 \mathrm{~Hz}$.

average $40 \mathrm{~dB}$ above the background, these pickups do not result in noticeable image disturbances.

By placing a plane mirror in the focal plane of lens 2, imaging of transmissive targets becomes possible. The sample is located directly in front of the mirror within the range of focal depth. Figure 5(a) depicts the phase-integrated transmission image of a high-density polyethylene (HDPE) plate with several defects such as soldering debris on top, a hole in the middle, and a scratch beneath. The fringes in the image are due to Fabry-Pérot interferences between mirror and sample. Figure 5(b) depicts another HDPE plate in which the letters "THz QCL" have been inscribed using a knife. The results demonstrate that the system is especially sensitive to the defects in THz-transparent materials. While imaging of reflective targets requires the precise angular alignment of the sample for a sufficient feedback level, in transmission mode such a limitation is not present. Hence, the full realtime potential of the setup can be exploited in this configuration.

In summary, we demonstrated the potential of the selfmixing effect in QCLs for real-time $\mathrm{THz}$ imaging in reflection and transmission modes. Fast image scans are achieved
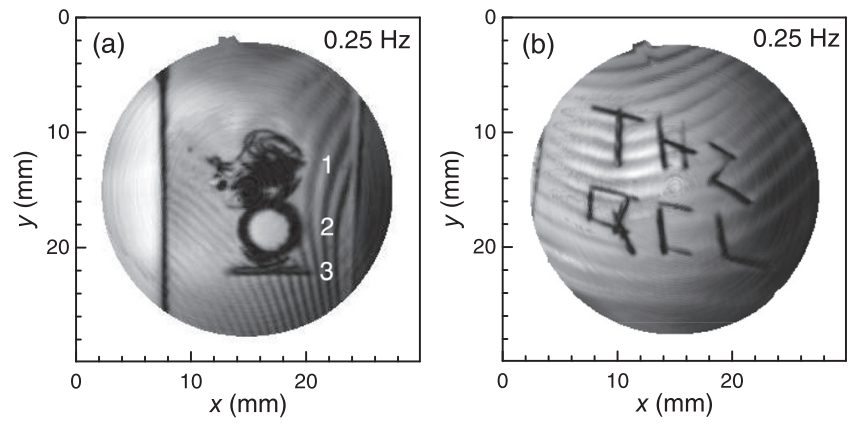

FIG. 5. (a) Transmission image of a damaged HDPE plate. 1: soldering debris, 2: hole, and 3: scratch. (b) Transmission image of a HDPE plate with inscribed letters. by deflecting the $\mathrm{THz}$ beam with a scanning mirror. By additionally modulating the laser frequency, amplitude and phase information can be separated. Since no parameter fitting is involved, this process can be performed in real time as well. For reflective samples, the former quantity corresponds to the power reflectivity and the latter to the depth-profile as well as the reflective phase shift of the sample. However, a quantitative analysis will require a dedicated calibration procedure to account for illumination inhomogeneities and the non-planar phase front of the scanned THz beam. At higher frame rates, the spatial resolution for a given field of view is currently limited by the mechanical inertia of the employed scanning mirror. Performance improvements are expected when using a system with two independent mirrors. Due to the sensitivity of the self-mixing effect and the ps response times of THz QCLs, such a system is expected to allow for high-resolution coherent imaging at video frame rates.

Part of this work was supported by the Deutsche Forschungsgemeinschaft. T.H. acknowledges the support by the Helmholtz Research School on Security Technologies.

${ }^{1}$ P. Dean, A. Valavanis, J. Keeley, K. Bertling, Y. L. Lim, R. Alhathlool, A. D. Burnett, L. H. Li, S. P. Khanna, D. Indjin et al., J. Phys. D: Appl. Phys. 47, 374008 (2014).

${ }^{2}$ A. D. Rakić, T. Taimre, K. Bertling, Y. L. Lim, P. Dean, D. Indjin, Z. Ikonić, P. Harrison, A. Valavanis, S. P. Khanna et al., Opt. Express 21, 22194 (2013).

${ }^{3}$ F. P. Mezzapesa, L. L. Columbo, M. Brambilla, M. Dabbicco, M. S. Vitiello, and G. Scamarcio, Appl. Phys. Lett. 104, 041112 (2014).

${ }^{4}$ P. Dean, A. Valavanis, J. Keeley, K. Bertling, Y. L. Lim, R. Alhathlool, S. Chowdhury, T. Taimre, L. H. Li, D. Indjin et al., Appl. Phys. Lett. 103, 181112 (2013).

${ }^{5}$ P. Dean, Y. L. Lim, A. Valavanis, R. Kliese, M. Nikolić, S. P. Khanna, M. Lachab, D. Indjin, Z. Ikonić, P. Harrison et al., Opt. Lett. 36, 2587 (2011).

${ }^{6}$ Y. Ren, R. Wallis, D. S. Jessop, R. Degl'Innocenti, A. Klimont, H. E. Beere, and D. A. Ritchie, Appl. Phys. Lett. 107, 011107 (2015).

${ }^{7}$ N. Rothbart, H. Richter, M. Wienold, L. Schrottke, H. T. Grahn, and H.W. Hübers, IEEE Trans. Terahertz Sci. Technol. 3, 617 (2013). 
${ }^{8}$ H. Richter, M. Greiner-Bär, S. G. Pavlov, A. D. Semenov, M. Wienold, L. Schrottke, M. Giehler, R. Hey, H. T. Grahn, and H.-W. Hübers, Opt. Express 18, 10177 (2010).

${ }^{9}$ M. Wienold, L. Schrottke, M. Giehler, R. Hey, W. Anders, and H. T. Grahn, Electron. Lett. 45, 1030 (2009).

${ }^{10}$ C. H. Henry, IEEE J. Quantum Electron. 18, 259 (1982).

${ }^{11}$ M. Lerttamrab, S. L. Chuang, C. Gmachl, D. L. Sivco, F. Capasso, and A. Y. Cho, J. Appl. Phys. 94, 5426 (2003).

${ }^{12}$ R. Lang and K. Kobayashi, IEEE J. Quantum Electron. 16, 347 (1980).
${ }^{13}$ G. Giuliani, M. Norgia, S. Donati, and T. Bosch, J. Opt. A: Pure Appl. Opt. 4, S283 (2002).

${ }^{14}$ R. P. Green, J.-H. Xu, L. Mahler, A. Tredicucci, F. Beltram, G. Giuliani, H. E. Beere, and D. A. Ritchie, Appl. Phys. Lett. 92, 071106 (2008).

${ }^{15}$ Y. Yu, G. Giuliani, and S. Donati, IEEE Photonics Technol. Lett. 16, 990 (2004).

${ }^{16}$ Y. L. Lim, P. Dean, M. Nikolić, R. Kliese, S. P. Khanna, M. Lachab, A. Valavanis, D. Indjin, Z. Ikonić, P. Harrison et al., Appl. Phys. Lett. 99, 081108 (2011). 